\title{
A NEW SOLUTION FOR 3D CRACK EXTENSION BASED ON LINEAR ELASTIC STRESS FIELDS
}

\author{
Y. H. Tai, M. W. Brown and J. R. Yates \\ Dept of Mechanical Engineering, University of Sheffield, UK. \\ E-mail:y.tai@sheffield.ac.uk
}

\begin{abstract}
A solution to the 3D stress field based on the maximum tangential stress (MTS) criterion is presented in this paper. The solution allows for the estimation of the critical crack plane, the direction of growth in terms of both twist and tilt angles and the equivalent crack driving force for a given mixed-mode loading condition. It also shows the graphical relationship between the three different stress intensities for a given driving force. Initial results have shown good correlation with experimental data obtained from literature.
\end{abstract}

\section{INTRODUCTION}

The principles of linear elastic fracture mechanics (LEFM) are founded on elastic material behaviour whereby the relationship between stress-strain is linear and only very small scale yielding occurs. Basic LEFM stress field equations derived from Cauchy stress tensors describe the stress field near a crack tip as function of location, 3D loading applied and geometry of the structure. By incorporating the stress intensity factor $(K)$ which takes into account the loading and geometry, the equations can be simplified to describe the magnitude of the stress field in terms of $K$ and location characterised by polar coordinates from the crack tip.

Based on the stress field equations formulated for the three fracture modes, this paper proposes an analytical solution to derive the crack path plane, growth direction and equivalent $K$ value. This utilises the $K_{\mathrm{I}}, K_{\mathrm{II}}$ and $K_{\text {III }}$ stress field equations in conjunction with the maximum tangential stress (MTS) criterion [1] which states that a crack will initiate in the direction where the largest circumferential stress at a small distance from the crack tip reaches a critical tensile value. The solution uses a tensor transformation to find the critical plane and direction of crack growth which then allows for the prediction of crack growth direction for a 3D case and, in turn, the equivalent crack driving force. 
The numerical results obtained for the MTS criterion show the graphical relationships between the applied stress intensities ( $K_{\mathrm{I}}, K_{\mathrm{II}}$ and $\left.K_{\mathrm{III}}\right)$ and the crack growth direction angle, the crack plane orientation and the crack driving force. The results are compared with both experimental and numerical data from literature and they are found to work well for predicting the crack plane and the equivalent driving force $K_{\text {eq, }}$, for materials that experience tensile mode fatigue cracking and fracture.

\section{ANALYTICAL SOLUTION}

The asymptotic expansion of the stress field around a crack tip in an isotropic linear elastic body was first proposed by Williams [2] and the first term of the expansion can be expressed as follows [3]:

$$
\begin{aligned}
& \sigma_{x x}=\frac{K_{I}}{\sqrt{2 \pi r}} \cos \frac{\theta}{2}\left(1-\sin \frac{\theta}{2} \sin \frac{3 \theta}{2}\right)-\frac{K_{I I}}{\sqrt{2 \pi r}} \sin \frac{\theta}{2}\left(2+\cos \frac{\theta}{2} \cos \frac{3 \theta}{2}\right) \\
& \sigma_{y y}=\frac{K_{I}}{\sqrt{2 \pi r}} \cos \frac{\theta}{2}\left(1+\sin \frac{\theta}{2} \sin \frac{3 \theta}{2}\right)+\frac{K_{I I}}{\sqrt{2 \pi r}} \sin \frac{\theta}{2} \cos \frac{\theta}{2} \cos \frac{3 \theta}{2} \\
& \sigma_{z z}=v\left(\sigma_{x x}+\sigma_{y y}\right) \\
& \sigma_{x y}=\frac{K_{I}}{\sqrt{2 \pi r}} \cos \frac{\theta}{2} \sin \frac{\theta}{2} \cos \frac{3 \theta}{2}+\frac{K_{I I}}{\sqrt{2 \pi r}} \cos \frac{\theta}{2}\left(1-\sin \frac{\theta}{2} \sin \frac{3 \theta}{2}\right) \\
& \sigma_{x z}=\frac{K_{I I I}}{\sqrt{2 \pi r}}\left(-\sin \frac{\theta}{2}\right) \\
& \sigma_{y z}=\frac{K_{I I I}}{\sqrt{2 \pi r}} \cos \frac{\theta}{2}
\end{aligned}
$$


Poisson's ratio $v$, is taken as 0.3 in these calculations. The $y$-direction is normal to the crack, and the crack extends in the positive $x$-direction if it remains coplanar.

The stresses from the expansion above are then grouped together to form a modified stress tensor $S$, where stress is multiplied by $\sqrt{ }(2 \pi r)$ to model the elastic singularity.

$S=\sqrt{2 \pi r}\left[\begin{array}{lll}\sigma_{x x} & \tau_{x y} & \tau_{x z} \\ \tau_{x y} & \sigma_{y y} & \tau_{y z} \\ \tau_{x z} & \tau_{y z} & \sigma_{z z}\end{array}\right]$

The modified stress tensor $S$ is then transformed from one set of Cartesian coordinates to another by means of rotation through two angles, to tilt and twist the crack by undergoing the matrix transformation:

$S^{\prime}=L S L^{t}$

where the tensor $L$ contains the direction cosines expressed in matrix form as follows:

$L=\left[\begin{array}{ccc}\cos \chi & \sin \chi & 0 \\ -\sin \chi \cos \psi & \cos \chi \cos \psi & \sin \psi \\ \sin \psi \sin \chi & -\sin \psi \cos \chi & \cos \psi\end{array}\right]$

where $\chi=$ tilt angle and $\psi=$ twist angle as illustrated in Figure 1 . The transformed stress tensor can then be expressed as:

$S^{\prime}=\sqrt{2 \pi r}\left[\begin{array}{ccc}\sigma_{x^{\prime} x^{\prime}} & \tau_{x^{\prime} y^{\prime}} & \tau_{x^{\prime} z^{\prime}} \\ \tau_{x^{\prime} y^{\prime}} & \sigma_{y^{\prime} y^{\prime}} & \tau_{y^{\prime} z^{\prime}} \\ \tau_{x^{\prime} z^{\prime}} & \tau_{y^{\prime} z^{\prime}} & \sigma_{z^{\prime} z^{\prime}}\end{array}\right]$

Here, $y^{\prime}$ is normal to the new crack plane and $x^{\prime}$ denotes the crack growth direction. 
In the first iteration of the analytical solution described in this paper, the maximum tangential stress (MTS) criterion $\left(\sigma_{y^{\prime} y^{\prime}}\right)$ was used as the failure criteria and the equations were solved to obtain the biggest possible value which corresponds to $\sigma_{y^{\prime} y^{\prime}}$ in the $S^{\prime}$ matrix. In this work, a value of $\mathrm{K}_{\mathrm{eq}}=10 \mathrm{MPa} \sqrt{\mathrm{m}}$ was used together with selected values of $\mathrm{K}_{\mathrm{II}}$ and $\mathrm{K}_{\mathrm{III}}$ from which $\mathrm{K}_{\mathrm{I}}, \chi$ and $\psi$ were then calculated. The tilt angle $\chi$ defines the crack growth direction along $x^{\prime}$ and the twist angle $\psi$ denotes the crack plane which is the plane of facets in a "factory roof" formation along the original crack front $z$. The results obtained are shown in the following section.
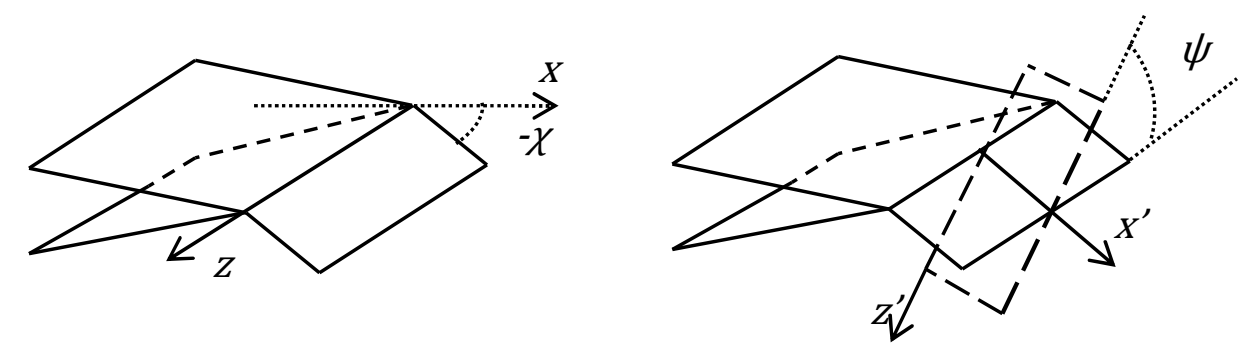

Figure 1: Schematic illustration of the negative tilt $(\chi)$ and subsequent twist $(\psi)$ angle

\section{RESULTS}

\section{New solution obtained from MTS criterion}

The results obtained from solving the analytical solution discussed above are presented in this section. Figure 2 illustrates the three dimensional surface plot of $\mathrm{K}_{\mathrm{l}}$, represented in the vertical z-axis, for a given $\mathrm{K}_{\mathrm{II}}, \mathrm{K}_{\mathrm{III}}$ and $\mathrm{K}_{\mathrm{eq}}=10$. If a $2 \mathrm{D}$ slice is taken for $\mathrm{K}_{\mathrm{I}}$ against $\mathrm{K}_{\mathrm{II}}$ with $\mathrm{K}_{\mathrm{III}}$ equal to zero, the relationship between $\mathrm{K}_{\mathrm{I}}$ and $\mathrm{K}_{\mathrm{II}}$ is approximately parabolic. If the same is done for $\mathrm{K}_{\mathrm{I}}$ and $\mathrm{K}_{\mathrm{III}}$, the relationship is rather linear if $\mathrm{K}_{\mathrm{III}}$ is the dominant term but becomes parabolic once $\mathrm{K}_{\mathrm{I}}$ becomes the dominant term (see Figure 5).

Figure 2 shows one quarter of the dome-shape failure surface, which is symmetrical with $K_{I I}$ and $K_{I I I}$, both of which can take negative values. Only positive values of $K_{I}$ have been used assuming crack closure will occur where negative $\mathrm{K}_{\mathrm{I}}$ values are calculated. 


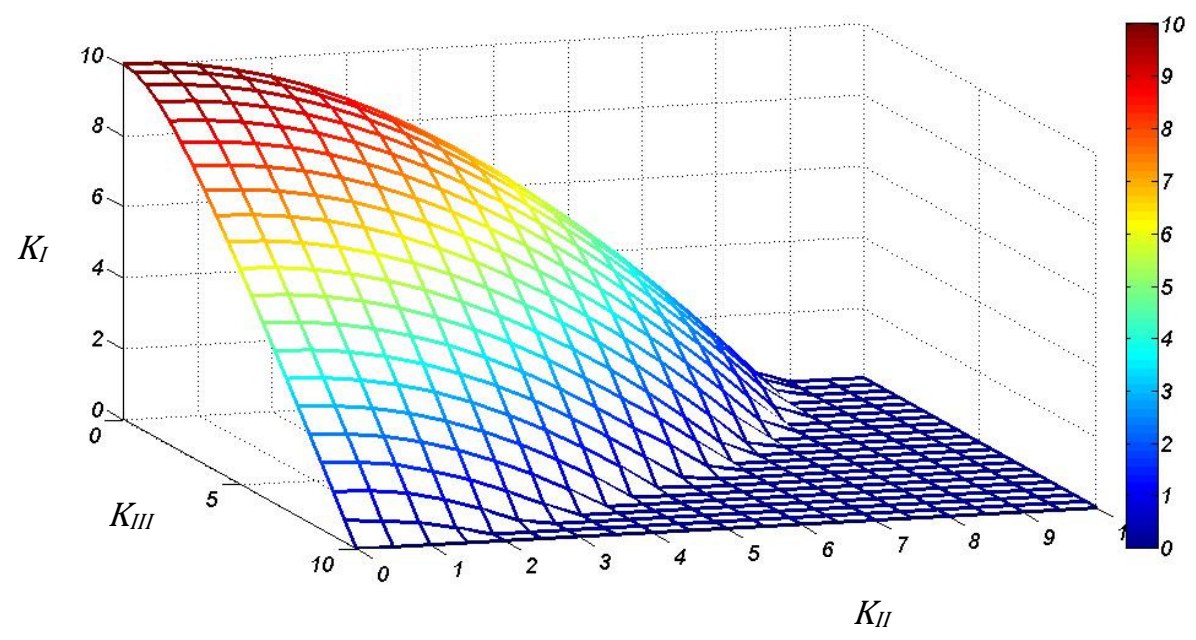

Figure 2: Illustration of the 3D stress intensity fields for $K_{e q}=10$

Figure 3 and Figure 4 show the change in the tilt $(\chi)$ and twist $(\psi)$ angle respectively for a given combination of $\mathrm{K}_{\mathrm{II}}$ and $\mathrm{K}_{\mathrm{III}}$. The results obtained from the tilt angle show that it is predominantly influenced by the $\mathrm{K}_{\text {II }}$ term and the $\mathrm{K}_{\mathrm{III}}$ term has only a small influence on the change in tilt angle. The opposite is true for the twist angle where the predominant influence is the $\mathrm{K}_{\text {III }}$ term although $\mathrm{K}_{\mathrm{II}}$ does have a more significant influence on the twist angle if compared to the influence of $\mathrm{K}_{\mathrm{III}}$ in the tilt angle. These surfaces are truncated where the calculated $\mathrm{K}_{\mathrm{I}}$ values have begun to turn negative.

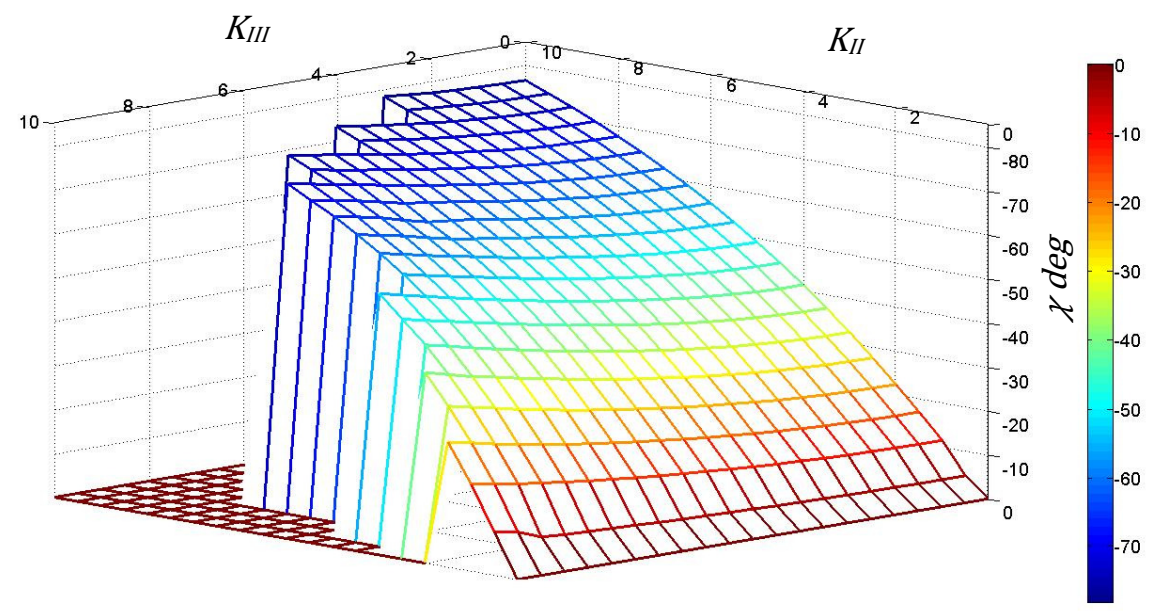

Figure 3: Change in tilt angle $\chi$ in relation to $K_{I I}$ and $K_{I I I}$ for $K_{e q}=10$ 


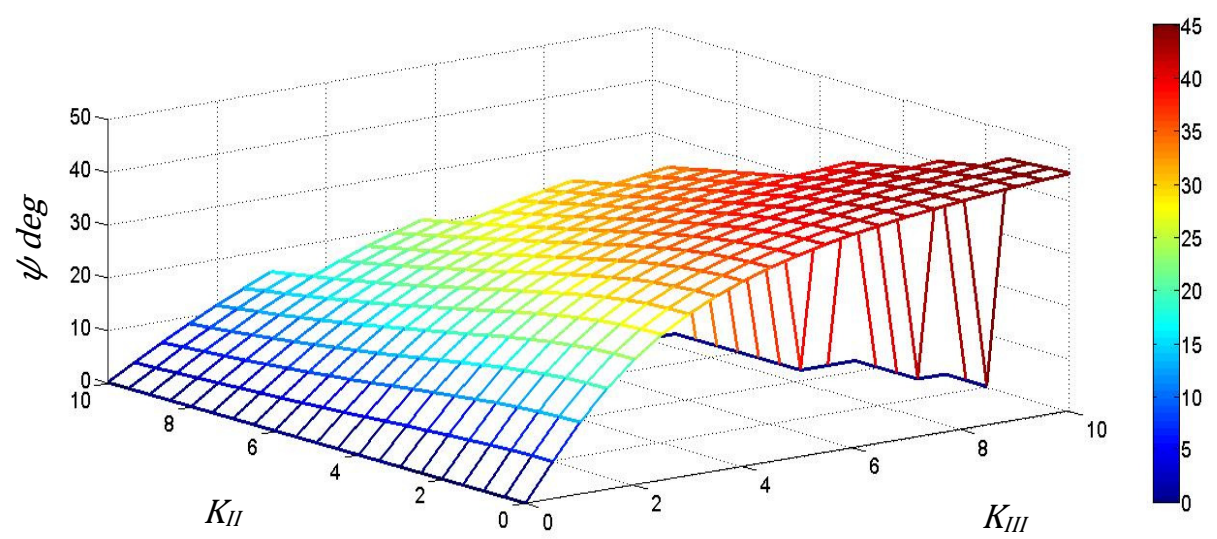

Figure 4: Change in twist angle $\psi$ in relation to $K_{\text {II }}$ and $K_{\text {III }}$ for $K_{e q}=10$

The maximum tilt angle for pure mode II was $70.5^{\circ}$ which differs slightly from results obtained in a solution proposed by Richard et al [4] who found the maximum tilt angle to be approximately $70.0^{\circ}$ using the same MTS criterion. Higher tilt angles can be achieved with negative nominal $\mathrm{K}_{\mathrm{I}}$ if cyclic crack closure conditions generate small negative $\mathrm{K}_{\mathrm{I}}$ values. In pure mode III, both the criterion in this paper and from literature [4] produced a maximum twist angle of $45.0^{\circ}$.

There are some further small discrepancies between these results and the two solutions of Richard [4] and Schollman [5] especially in the calculated tilt and twist angle. This could be due to assumptions made in the solutions proposed by Richard and Schollman which use Mohr's circle to obtain the angle required to satisfy the MTS criterion. The use of a 2D Mohr's circle provides only an approximate solution for a 3D stress field. The only assumption made in the solution in this paper is that the local stress intensity is equal to $\mathrm{K}_{\text {eq. }}$. Further detailed analysis is required to determine the exact regions of the discrepancies and which provides the better solution to the 3D stress fields.

\section{Comparison with experimental data from literature}

Figure 5 shows the calculated failure loci based on the MTS criterion which was used in this solution The experimental data seen in the plot were obtained from literature [6]. If the $\mathrm{K}_{\mathrm{I}}$ and $\mathrm{K}_{\text {III }}$ values given in literature are normalised by $\mathrm{K}_{\text {Ith }}$, the plots show that the failure loci underestimates slightly the initiation of cracking under near threshold loading conditions. If the $\mathrm{K}_{\mathrm{Ith}}$ is increased by approximately 12 percent to 
account for crack closure induced by roughness in mixed mode of the fracture surface [7], the results show that both experimental data and position of the failure loci agree very well. Further data can be obtained from literature [7-9] which shows that the MTS criterion works well for correlation of failure under mixed mode I + II loading conditions.

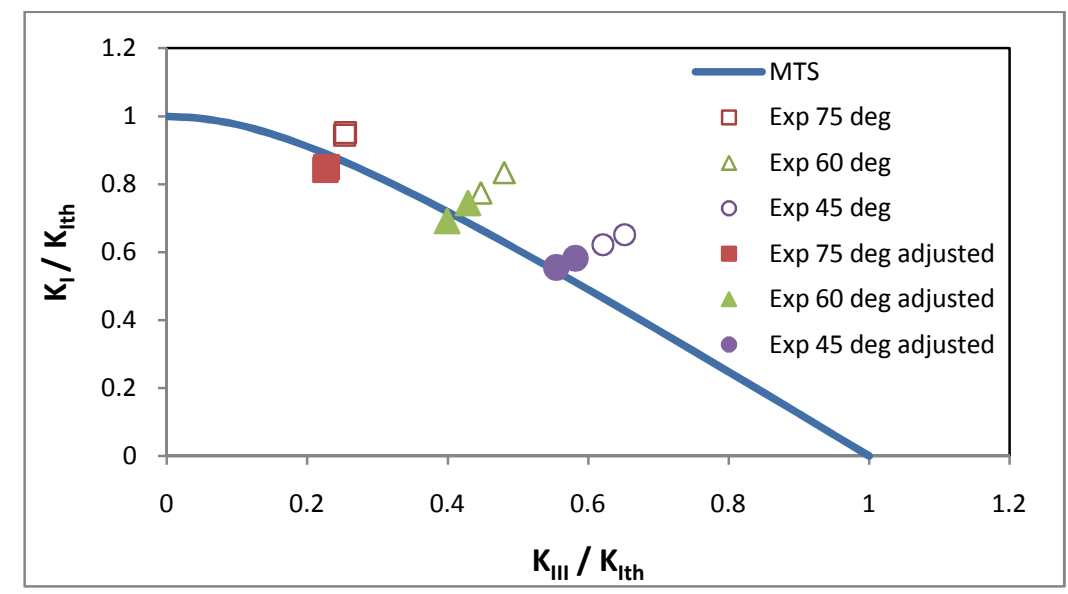

Figure 5: Failure loci for mixed mode (I + III) loading for tests of Pook [10] on En 3 steel

Further analysis of the results shows that the twist angle predicted for mixed mode $\mathrm{K}_{\mathrm{I}}+\mathrm{K}_{\mathrm{III}}$ loading correlates with the experimental data from literature [6] used in this paper. Figure 6 shows the comparison between the predicted twist angle needed for crack propagation and the initial slit angle used in the experiments. The discrepancies in the estimates are in the region of $\pm 5.0^{\circ}$ but further work is needed on both the experimental and analytical front to determine where the errors originate. More precise readings of the facet angles for individual cracks are needed to relate to $\psi$. Crack angles for $\chi$, for mixed mode I + II have been reported in various papers in literature [7-9] indicating good correlation to the MTS criterion.

Calculations of crack driving force, propagation plane and growth direction are required in the analysis of failure of rails under rolling contact for high speed train lines. The non-proportional loading history experienced introduces modes I, II and III to the problem. Similar non-proportional stresses are found in automotive suspension and drivetrain components due to the complexities of service load histories. 


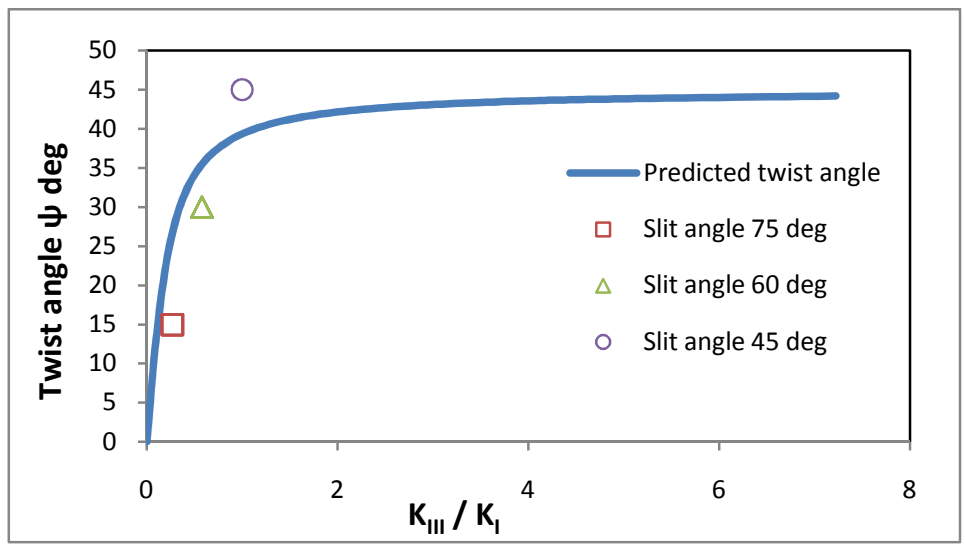

Figure 6: Comparison between predicted and experimental twist angle $\psi$ for mixed mode $(I+I I I)$ loading

\section{FINAL COMMENTS}

An analytical solution for predicting the parameters which describe 3D crack extension under mixed-mode loading is presented in this paper. The solution allows for the calculation of crack growth direction for a 3D case and in turn the equivalent crack driving force. At the moment, the solution is based on the MTS criterion and comparison of the results with experimental data from literature has shown good correlation. Further work is underway for more detailed mixed mode I + II + III results and further comparisons with experimental data. The solution is also being extended for a different failure criterion of maximum shear stress to correlate shear mode cracks.

\section{REFERENCES}

[1] Erdogan F and Sih G C, On the crack extension in plates under plane loading and transverse shear. Journal of Basic Engineering, 1963. 85: p. 525-527.

[2] Williams M L, On the stress distribution at the base of a stationary crack. Journal of Applied Mechanics, 1957. 24: p. 109-114.

[3] Miannay D P, Fracture Mechanics. Mechanical Engineering Series, ed. Ling F F. 1997: Springer. 337. 
[4] Richard H A, Fulland M, and Sander M, Theoretical crack path prediction. Fatigue and Fracture of Engineering Materials and Structures, 2004. 28: p. 312.

[5] Schollman M, Richard H A, Kullmer G, and Fulland M, A new criterion for the prediction of crack development in multiaxially loaded structures. International Journal of Fracture, 2002. 117: p. 129-141.

[6] Yates J R, Fatigue thresholds under mixed-mode (I+III) loading. International Journal of Fatigue, 1991. 5: p. 383-388.

[7] Tong J, Yates J R, and Brown M W, The formation and propagation of Mode I branch cracks in mixed mode fatigue failure. Engineering Fracture Mechanics, 1997. 56(2): p. 213-231.

[8] Smith D J, Ayatollahi M R, and Pavier M J, The role of T-stress in brittle fracture for linear elastic materials under mixed-mode loading. Fatigue and Fracture of Engineering Materials and Structures, 2000. 24: p. 137-150.

[9] Baloch R A and Brown M W. Crack closure analysis for the threshold of fatigue crack growth under mixed-mode I/II loading. in Mixed-Mode Fatigue and Fracture. 1993. Vienna: Mechanical Engineering Publications.125-137

[10] Pook L P, The fatigue crack direction and threshold behaviour of mild steel under mixed mode I and III loading. International Journal of Fatigue, 1985. 7: p. 21-30. 